\title{
Evaluación de la cultura sobre seguridad del paciente entre médicos residentes de Medicina familiar y comunitaria en un servicio de urgencias hospitalario
}

\section{Evaluation of patient safety culture among family and community medicine residents in a hospital $A$ and $E$ department}

\author{
C. Jaraba Becerril ${ }^{1}$, M.T. Sartolo Romeo' ${ }^{1}$, M.V. Villaverde Royo ${ }^{2}$, L. Espuis Albas ${ }^{3}$, \\ M. Rivas Jiménez ${ }^{4}$
}

\section{RESUMEN}

Fundamento. La mejora de la seguridad de los pacientes es prioritaria en los centros sanitarios. En los programas de las especialidades no aparece como un área de aprendizaje específica. El objetivo de este trabajo es evaluar la cultura de seguridad de los MIR de Medicina de Familia.

Material y métodos. Estudio descriptivo transversal. Como cuestionario se utilizó la versión española del "Hospital Survey on Patient Safety". Se determinaron las fortalezas y oportunidades de mejora del servicio de Urgencias.

Resultados. La dimensión mejor valorada fue el trabajo en equipo en urgencias. Las peor calificadas fueron "dotación de personal" y "apoyo de la gerencia". Ningún encuestado había notificado incidentes en el servicio.

Conclusiones. Existe, entre los MIR encuestados, un desconocimiento de las vías para notificar eventos adversos, así como una deficiencia formativa en cultura de seguridad. Ésta debería potenciarse en la formación de los especialistas en Medicina Familiar y Comunitaria.

Palabras clave. Seguridad del paciente. Servicio de Urgencias. Médicos residentes. Encuesta.

\begin{abstract}
Background. Improving patient safety is a priority in all health care centres. It does not appear as a specific area of training in specialty programs. The aim of this study is to evaluate the safety culture of Family Medicine residents (MIR - Médico Interno Residente/Medical Internship and Residency).
\end{abstract}

Methods. Transversal descriptive study. The Spanish version of the "Hospital Survey on Patient Safety" questionnaire was used. Strengths and opportunities for improving the service of the A \& E Department were determined.

Results. The most highly valued dimension was teamwork in the A \& E Department. The worst qualified were "staffing" and "management support". None of those surveyed had notified any incidents in the unit.

Conclusions. Amongst the MIR surveyed there is a lack of knowledge about the channels for notifying adverse events, as well as a training deficiency in safety culture. This should be strengthened in the training of specialists in Family and Community Medicine.

Key words. Patient safety. A \& E Department. Internship and residency. Survey. United States Agency for Healthcare Research and Quality.
1. Médico de Familia. Centro de Salud Albarracín. Teruel.

2. Unidad Docente Medicina Familiar y Comunitaria Sector Zaragoza III y Calatayud. Profesor asociado Facultad de Ciencias de la Salud de la Universidad San Jorge.

3. Médico Adjunto del Servicio de Urgencias. Hospital de Viladecans. Barcelona.

4. Jefe y Coordinador del Servicio de Urgencias. Hospital Clínico Universitario Lozano Blesa. Zaragoza. Jefe de Estudios MFC Sector Zaragoza III y Calatayud.

\section{Correspondencia:}

Carlos Jaraba Becerril

Centro de Salud Albarracín

Camino del Santo Cristo, $\mathbf{s} / \mathrm{n}$

44100 Albarracín

Teruel

cjaraba@hotmail.com

Recepción: 25 de marzo de 2013

Aceptación provisional: 3 de junio de 2013

Aceptación definitiva: 13 de junio de 2013 


\section{INTRODUCCIÓN}

Actualmente, la mejora de la seguridad de los pacientes (SP) se ha convertido en una necesidad y en objetivo prioritario en los programas de salud. En los centros sanitarios se realizan protocolos y se ejecutan medidas para evitar los incidentes o eventos adversos. Sin embargo, en los planes formativos de las especialidades médicas en España no queda recogido como un área de aprendizaje específica ${ }^{1}$.

La mayoría de los médicos internos residentes (MIR) realizan parte de su labor asistencial en las urgencias hospitalarias. De todos ellos, un alto porcentaje pertenecen a la especialidad de Medicina Familiar y Comunitaria.

En el estudio EVADUR realizado en $2010^{2}$, se determinó que el $12 \%$ de los pacientes que en España acuden a un servicio de urgencias sufren algún tipo de evento adverso. Esto está condicionado, entre otros, por factores como la sobrecarga asistencial, el estrés, la fatiga, la falta de experiencia y la complejidad de los pacientes.

Con este trabajo se pretende conocer cuál es la percepción sobre SP que tienen los MIR de Medicina Familiar y Comunitaria en un hospital docente de tercer nivel.

\section{MATERIAL Y MÉTODOS}

Se trata de un estudio descriptivo transversal, realizado mediante cuestionario autoadministrado, durante el segundo trimestre de 2011. Los participantes fueron los 52 facultativos MIR de Medicina Familiar y Comunitaria pertenecientes a la Unidad Docente de Medicina Familiar y Comunitaria Zaragoza III. Todos ellos realizaron parte de su tarea asistencial en el servicio de Urgencias del Hospital Clínico Universitario Lozano Blesa de Zaragoza. Tras un segundo recordatorio se obtuvieron 44 cuestionarios debidamente cumplimentados, lo que supone una tasa de respuesta del $84,6 \%$.

Para evaluar la cultura sobre seguridad del paciente que tienen estos profesionales, se utilizó la versión española del Hospital Survey on Patient Safety. Éste es un cuestionario que adapta al contexto español la encuesta original de la Agency for
Healthcare Research and Quality (AHRQ) y fue realizado por la Agencia de Calidad del Ministerio de Sanidad y Consumo y la Universidad de Murcia en el año 20053. Mediante 42 preguntas, la encuesta proporciona información relativa a las doce dimensiones de la cultura de seguridad que se exponen en la tabla 1. Además, en la versión española de la encuesta existe un anexo con 9 ítems adicionales, así como una pregunta sobre la calificación global de la seguridad en el servicio donde trabaja el encuestado, y otra sobre el número de incidentes notificados en el último año.

Cada una de las 42 preguntas agrupadas en dimensiones cuenta con 5 posibles respuestas, según el grado de acuerdo o desacuerdo con cada ítem (Tabla 1). Para trabajar los datos con mayor facilidad, se agruparon en 3 categorías siguiendo la metodología de otros trabajos que han utilizado este cuestionario previamente (respuesta positiva, respuesta neutral y respuesta negativa) $)^{4,5}$.

Con los resultados calculados, se determinaron las fortalezas y debilidades, $\mathrm{u}$ oportunidades de mejora del servicio de urgencias, siguiendo los siguientes criterios:

Se clasificó una variable o una dimensión como fortaleza cuando:

- El porcentaje fue mayor o igual a $75 \%$ de respuestas positivas a preguntas formuladas en positivo.

- El porcentaje fue mayor o igual a $75 \%$ de respuestas negativas a preguntas formuladas en negativo.

Para clasificar un ítem o una dimensión como oportunidad de mejora se emplearon los siguientes criterios:

- Porcentaje mayor o igual a 50\% de respuestas negativas a preguntas formuladas en positivo.

- Porcentaje mayor o igual a 50\% de respuestas positivas a preguntas formuladas en negativo.

Para el análisis estadístico se empleó el programa SPSS versión 15.0, realizándose un análisis descriptivo de las frecuencias y frecuencias acumuladas de los datos aportados por los residentes aplicando la metodología de la AHRQ y su adaptación española que figura en el documento del Ministerio ${ }^{3}$. 
Tabla 1. Dimensiones sobre la cultura de seguridad.

\section{Dimensiones sobre la cultura de seguridad}

1. Frecuencia de eventos notificados (3 ítems).

2. Percepción de seguridad (4 ítems).

3. Expectativas y acciones de la dirección del servicio que favorecen la seguridad (4 ítems).

4. Aprendizaje organizacional. Mejora continua (3 ítems).

5. Trabajo en equipo en el servicio (4 ítems).

6. Franqueza en la comunicación (3 ítems).

7. Feedback y comunicación sobre errores (3 ítems).

8. Respuesta no punitiva a los errores (3 ítems).

9. Dotación de personal (4 ítems).

10. Apoyo de la gerencia del hospital a la seguridad del paciente (3 ítems).

11. Trabajo en equipo entre unidades (4 ítems).

12. Problemas en los cambios de turno y en las transiciones entre unidades y servicios (4 ítems).

Posibles respuestas a cada ítem

- "Muy en desacuerdo" / "Nunca"

- "En desacuerdo" / "Casi nunca"

- "Indiferente" / "A veces"

- "De acuerdo" / "Casi siempre"

- "Muy de acuerdo" / "Siempre"

\section{RESULTADOS}

Con una tasa de respuesta del $84,6 \%$, se obtuvieron 44 cuestionarios adecuadamente cumplimentados. En dos de ellos quedó uno de los ítems sin respuesta y en otro quedaron dos preguntas sin responder. El resto contestaron a todas las preguntas formuladas en la encuesta.

Clasificando nuestros resultados en fortalezas y oportunidades de mejora, según los criterios anteriormente expuestos, nos encontramos los siguientes datos: la variable "El personal se apoya mutuamente" apareció como única fortaleza con una tasa de respuestas positivas del $81,8 \%$, y no existiendo ninguna respuesta negativa en esta cuestión. Las variables que se presentaron como oportunidades de mejora por su alta tasa de respuestas de carácter negativo quedan reflejadas en la tabla 2.
En cuanto a los resultados por dimensiones, la mejor valorada hace referencia al "Trabajo en equipo dentro del servicio" con una tasa de respuestas positivas del $64,7 \%$. Ninguna otra de las dimensiones valoradas alcanzó un porcentaje de respuestas positivas del $50 \%$. La peor calificada fue "Dotación de personal" con un porcentaje del $68,1 \%$ de respuestas negativas y a la que solo un $15,9 \%$ respondieron con una opinión positiva. Con una alta proporción de respuestas negativas encontramos también "Apoyo de la gerencia del hospital en la seguridad del paciente" (54,9\% de respuestas negativas), "Problemas en cambios de turno y transiciones entre servicios" y "Trabajo en equipo entre servicios" con un $46 \%$ y un $40,9 \%$ respectivamente. La dimensión "Percepción de seguridad" también contó con una tasa de respuestas negativas del $40,9 \%$. 
Tabla 2. Porcentaje de respuestas contestadas de una manera negativa para la seguridad del paciente en el servicio de urgencias

\begin{tabular}{lc}
\hline \multicolumn{1}{c}{ Variables } & $\%$ \\
\hline A veces no se atiende adecuadamente al paciente porque la jornada laboral es agotadora. & 84,1 \\
\hline Trabajamos bajo presión para realizar demasiadas cosas demasiado deprisa. & 75,0 \\
\hline Las diferentes unidades del hospital no se coordinan bien. & 72,7 \\
\hline Hay suficiente personal para afrontar la carga de trabajo. & 70,5 \\
\hline La gerencia del hospital sólo parece interesarse por la seguridad del paciente cuando ya ha ocurrido & 70,5 \\
\hline algún suceso adverso en un paciente. & 56,8 \\
\hline La gerencia del hospital facilita un clima laboral que favorece la seguridad del paciente. & 54,5 \\
\hline Cuando se comete un error, el personal teme que eso quede en su expediente. & 52,3 \\
\hline Nunca se aumenta el ritmo de trabajo si eso implica sacrificar la seguridad del paciente. & 52,3 \\
\hline La información del paciente se pierde, en parte, cuando estos se transfieren de una unidad a otra. & 52,3 \\
\hline No se producen más fallos por casualidad. & 50,0 \\
\hline El personal puede cuestionar con total libertad las decisiones o acciones de sus superiores.
\end{tabular}

Es digno de destacar un elevado porcentaje de respuestas neutras en las dimensiones "Retroalimentación y comunicación sobre errores" (55,3\%), "Aprendizaje organizacional y mejora continua" con un $52,2 \%$ y "Franqueza en la comunicación" y "Frecuencia de eventos notificados" con un $51,9 \%$ y un $51,5 \%$ de respuestas neutras respectivamente.

El cuestionario contaba con una pregunta en la que se solicitaba a los encuestados que valorasen, en una escala del 1 al 10 , el grado de seguridad del paciente en el servicio de urgencias. Fue respondida en el $72,7 \%$ de los casos, obteniéndose una nota media de 5,9. Además se preguntaba a todos si en el último año habían notificado por escrito algún incidente. Ninguno de los MIR encuestados había notificado incidentes en el servicio de Urgencias.

En la versión española de la encuesta existe un anexo con información adicional. De las seis variables que se contestaron hay que destacar los resultados positivos de dos de ellas: "Antes de realizar una nueva prescripción se revisa el listado de medicamentos que está tomando el paciente" y "En los pacientes probablemente terminales, se indagan de forma anticipada sus prefe- rencias sobre tratamientos y procedimientos de soporte vital". La tasa de respuestas positivas fue de $88,6 \%$ y $75 \%$ respectivamente. Sin embargo, a la variable "Se elaboran informes o resúmenes de historias clínicas sin tener delante la documentación" sólo el $6,8 \%$ contestó nunca o casi nunca.

\section{DISCUSIÓN}

En la mayoría de los trabajos publicados en los que se valora el clima de SP en los servicios de urgencias, no se recoge de una manera específica la percepción que tienen los facultativos MIR sobre esta materia. Por lo tanto algunas de las diferencias que encontramos con otros trabajos similares podrían deberse a este hecho. Como limitación habría que decir que los datos se han recogido en un solo hospital y solo entre residentes de Medicina de Familia. En este sentido, conviene remarcar que la naturaleza cuantitativa del diseño empleado limita la interpretación de alguno de los resultados obtenidos, por lo que abre camino para futuros trabajos de base cualitativa (entrevistas) que complementen este estudio para mejorar la cultura de seguridad entre los MIR. 
En cuanto a los resultados obtenidos en este estudio, se debe destacar que entre las variables peor valoradas (Tabla 2) se encuentran varias que hacen referencia a la percepción de que no hay personal suficiente y que la carga de trabajo es excesiva. Según se recoge en las encuestas, esto no permite atender de una manera óptima a los pacientes de urgencias. Los residentes perciben que el ritmo de trabajo que hay en el servicio pone en peligro la seguridad de los pacientes.

Por dimensiones, las dos oportunidades de mejora encontradas son "Dotación de personal" y "Apoyo de la gerencia del hospi- tal en la seguridad del paciente”. Son también las peor valoradas en otros estudios ${ }^{5}$. En el diagrama de Pareto (Fig. 1) puede observarse que cuatro dimensiones de la cultura de seguridad son las responsables de más de la mitad de las respuestas negativas obtenidas en el estudio. Estas dimensiones son "Dotación de personal", "Problemas en los cambios de turno y transiciones entre servicios", "Percepción de seguridad", "Apoyo de la gerencia del hospital en la seguridad del paciente". Esto coincide con los resultados de estudios previos ${ }^{5,6}$, y se debe tener en cuenta a la hora de priorizar las medidas a tomar para mejorar la seguridad del paciente.

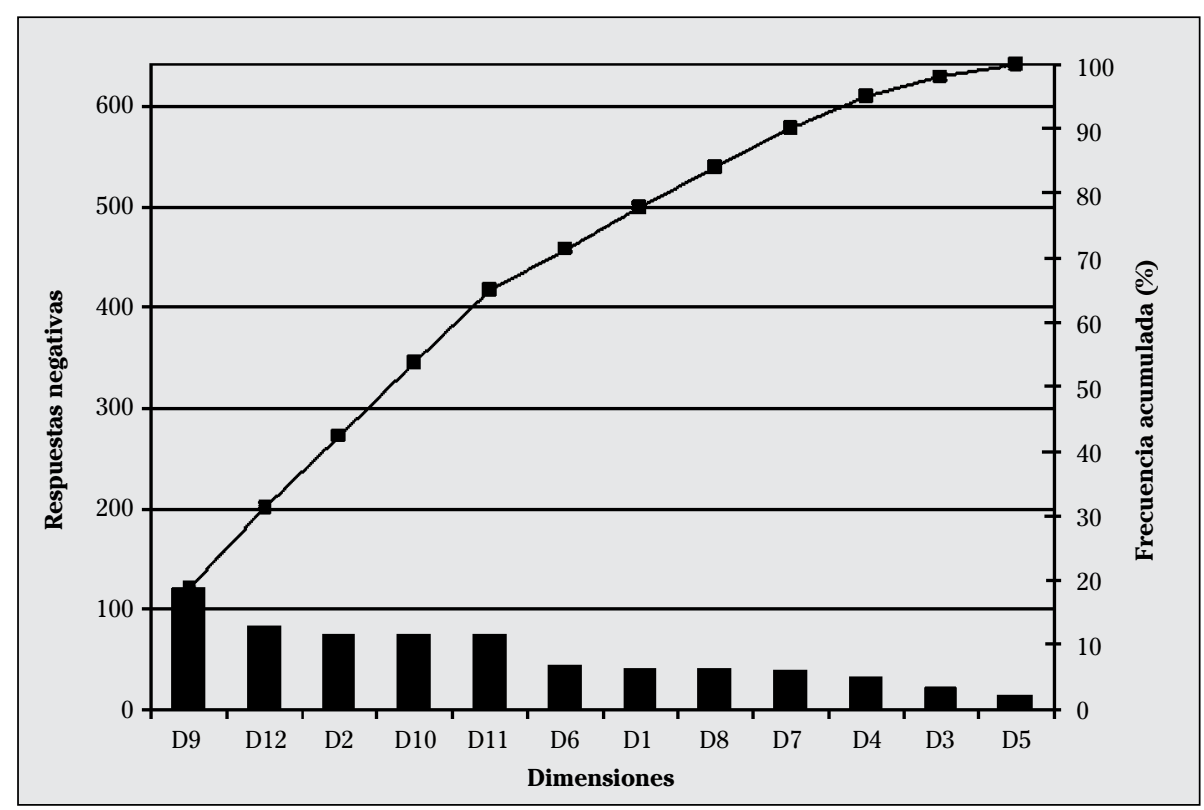

Figura 1. Oportunidades de mejora. Dimensiones: D9 Dotación de personal, D12 Problemas en los cambios de turno y en las transiciones entre unidades y servicios, D2 Percepción de seguridad, D10 Apoyo de la gerencia a la seguridad del paciente, D11 Trabajo en equipo entre unidades, D6 Franqueza en la comunicación, D1 Frecuencia de eventos notificados, D8 Respuesta no punitiva a los errores, D7 Feedback y comunicación sobre errores, D4 Aprendizaje organizacional/Mejora continua, D3 Expectativas y acciones de la dirección del servicio que favorecen la seguridad, D5 Trabajo en equipo en el servicio.

No se debe descuidar el alto porcentaje de respuestas neutras (superior al 50\%) en cuatro de las doce dimensiones sobre la SP. Tres de ellas ("Frecuencia de eventos notificados", "Retroalimentación y comunicación sobre errores" y "Aprendizaje organizacional y mejora continua") hacen referencia a la notificación de eventos y a los mecanismos de los que se dispone para encontrar sus causas, y así evitar que vuelvan a pro- 
ducirse. En nuestra opinión esto podría estar motivado por un desconocimiento, por parte de los MIR, de los procedimientos y mecanismos con los que cuenta el servicio de Urgencias para preservar la SP. Nos reafirma en ello el dato de que ninguno de los 44 médicos encuestados había notificado ningún incidente adverso en el último año.

La otra dimensión con elevado número de respuestas de carácter neutro, "Franqueza en la comunicación dentro del servicio", destaca además por presentar sólo un $15,9 \%$ de respuestas positivas. Se trata de un dato muy llamativo, y que además dista mucho de los datos obtenidos en otros estudios en los que, a diferencia de éste, no aparecen detalladamente los resultados de los MIR. En un estudio realizado por la Sociedad Española de Medicina de Urgencias y Emergencias (SEMES) en 2011, se obtuvo un $42 \%$ de respuestas positivas en esta dimensión ${ }^{5}$.

Es posible que el desconocimiento por parte de los médicos residentes de las vías para notificar incidentes, o para llevar a cabo acciones de mejora del servicio de Urgencias, sea la razón por la que los temas sobre SP no se tratan abiertamente. A nuestro juicio, esta última afirmación queda respaldada por la alta proporción de respuestas negativas en dos de las variables del estudio: "Cuando se comete un error el personal teme que eso quede en su expediente" y "El personal puede cuestionar con total libertad las decisiones o acciones de sus superiores". Esto puede hacer que se omita información necesaria para la mejora de la seguridad del paciente en el servicio.

Se debe tener en cuenta que es básico para conseguir avances en la SP generar un ambiente de confianza mutua con trabajadores motivados para la mejora del servicio. Para ello es necesario que se pueda opinar libremente sin temor a represalias y que el personal conozca las medidas y soluciones que se toman para mejorar la seguridad $^{7}$.

Resultan de interés documentos técnicos publicados en webs oficiales de servicios de Salud como las del Servicio de Urgencias del Complejo Donostia de San Sebastián ${ }^{8}$, que publica boletines en los que se explican detalles sobre seguridad del paciente para su difusión. Iniciativas como esta pueden ser un buen mecanismo para mejora la cultura de seguridad de nuestros médicos residentes.

En los últimos años los trabajos y los artículos sobre cultura de seguridad en los distintos ámbitos de la asistencia sanitaria se han multiplicado. Al mismo tiempo las sociedades médicas, e incluso el Ministerio de Sanidad, han realizado grandes encuestas para evaluar la cultura de seguridad y han proporcionado herramientas para mejorarla ${ }^{3-5}$. Sin embargo en los programas de las especialidades médicas ${ }^{9,10}$, y en concreto en el de Medicina Familiar y Comunitaria, la SP tan sólo aparece recogida de una manera tangencial, por lo que depende de cada unidad formativa el que se impartan conocimientos sobre la materia a sus residentes. Además no es una de las áreas de formación que más atractivo suele tener para los residentes, que suelen verlo como algo poco útil para su labor asistencial como especialistas ${ }^{11}$. Por eso habitualmente no buscan esta formación de una manera voluntaria.

Si desde los organismos gubernamentales, las sociedades médicas y los propios hospitales se pretende mejorar la cultura de seguridad, parece que debería potenciarse la formación de los MIR en este ámbito de conocimiento. Probablemente el modo de llevarlo a cabo sería su inclusión, de una manera clara, en los programas formativos de las diferentes especialidades.

\section{BIBLIOGRAFÍA}

1. SAURA J. La seguridad del paciente, un área competencial y una oportunidad formativa para los residentes de medicina familiar y comunitaria. Aten Primaria 2010; 42: 539-540.

2. Tomas S, Chanovas M, Roqueta F, Alcaraz J, ToRANZO T. EVADUR: eventos adversos ligados a la asistencia en los servicios de urgencias de hospitales españoles. Emergencias 2010; 22: 415-428.

3. Cuestionario sobre seguridad de los pacientes: versión española adaptada de Hospital Survey on Patient Safety. Madrid: Ministerio de Sanidad y Consumo; 2005. (Consultado el 4 de junio 2013). Disponible en:http://www. 
msc.es/organizacion/sns/planCalidadSNS/ docs/CuestionarioSeguridadPacientes1.pdf.

4. Análisis de la cultura sobre seguridad del paciente en el ámbito hospitalario del Sistema Nacional de Salud Español. Madrid: Ministerio de Sanidad y Política Social; 2009.

5. Roqueta E, Tomás S, Chanovas M. Cultura de seguridad del paciente en los servicios de urgencias: resultados de su evaluación en 30 hospitales del Sistema Nacional de Salud español. Emergencias 2011; 23: 356-364.

6. Saturno PJ, Da Silva ZA, De Oliveira-Sousa SL, Fonseca YA, De Souza-Oliveira. Grupo Proyecto ISEP. Med Clin (Barc) 2008; 131 (Supl 3): 18-25.

7. Tomás S, Gimena I. La seguridad del paciente en urgencias y emergencias. An Sist Sanit Navar 2010; 33 (Supl 1): 131-148.

8. Grupo de Seguridad [sede Web] ${ }^{*}$. Donostia: Servicio de Urgencias Hospital Donostia;
2010; [acceso 4 de junio de 2013]. Boletines Enero 2013-1. Disponible en: http://www.urgenciasdonostia.org/Portals/0/Organizacion/ Seguridad/Boletines/Enero\%202013-1.pdf

9. Orden SCO/1198/2005, de 3 de marzo, por la que se aprueba y publica el programa formativo de la especialidad de Medicina Familiar y Comunitaria. Boletín Oficial del Estado, núm. 150 (3 de mayo de 2005).

10. Orden SCO/227/2007, de 24 de enero, por la que se aprueba y publica el programa formativo de la especialidad de Medicina Interna. Boletín Oficial del Estado, núm. 33 (7 de febrero de 2007).

11. Vázquez G, Rodríquez M, Rubacabo L, García A, Murillo F, Navarrete $P$ et al. Los residentes en las unidades de cuidados intensivos, ¿cuál es su percepción de la formación que reciben? Educ Med 2011; 14: 189-194. 
